\title{
China Academic Library and Information System: An Academic Library Consortium in China
}

Since its inception in 1998, China Academic Library and Information System (CALIS) has become the most important academic library consortium in China. CALIS is centrally funded and organized in a tiered structure. It currently consists of thirteen management or information centers and seventy member libraries' 700,000 students. After more than a year of development in information infrastructure, a CALIS resource-sharing network is gradually taking shape.

ike their counterparts in other countries, academic libraries in China are facing such thorny problems as shrinking budgets, growing patron demands, and rising costs for purchasing books and subscribing to periodicals. It has thus become increasingly difficult for a single library to serve its patrons to their satisfaction. Under these circumstances, the idea of resource sharing among academic libraries was born.

Library consortia provide an organizational form for libraries to share their resources. The Georgia Library Learning Online (GALILEO), the Virtual Library of Virginia (VIVA), and OhioLINK are among the wellknown library consortia in the United States. ${ }^{1}$ Traditionally, the primary purpose of establishing a library consortium is to share physical resources such as books and periodicals among members. More recently, however, advances in computer, information, and telecommunication technologies have dramatically revolutionized the way in which information is acquired, stored, accessed, and transferred. Sharing electronic resources has rapidly become another important goal for library consortia.

\section{What Is CALIS?}

In May 1998, as one of the two public service systems in "Project 211," the China Academic Library and Information System (CALIS) project was approved by the State Development and Planning Commission of China after a two-year feasibility study by experts from academic libraries across the country.

CALIS is a nationwide academic library consortium. Funded primarily by the Chinese government, it is

Longji Dai is Director, Peking University Library, and Deputy Director, CALIS Administrative Center; Ling Chen is Deputy Director, CALIS Administrative Center; and Hongyang Zhang is Deputy Director, Reference Department, Peking University Library. intended to serve multiple resource-sharing functions among the participating libraries-including online searching, interlibrary loan, document delivery, and coordinated purchasing and cataloguing-by digitizing resources and developing an information service network.

\section{Structure and Management of CALIS}

A library consortium is an alliance formed by member libraries on a voluntary basis to facilitate resource sharing in pursuit of common interests. Whether a consortium can operate successfully depends in large part on how it is managed.

CALIS differs from library consortia in the United States in that it is a national network. It resembles multistate consortia in the United States with respect to geographic distribution of member libraries, but it is like tightly knit or even centrally funded statewide ones in terms of management. ${ }^{2}$

The CALIS members are distributed in twenty-seven provinces, cities, and autonomous regions in China, making an entirely centralized management difficult. After surveying some of the major library consortia in the United States, Europe, and Russia, CALIS adopted an organizational mode characterized by a combination of both centralized and localized management-that is, a three-tiered structure (figure 1).

In order to improve the management efficiency and maximize the sharing of various resources including funds, CALIS has established a coordination and management network comprising one national administrative center (which also serves as the North Regional Center), five national information centers (see table 1) and seven regional information centers (see table 2). The thirteen centers are maintained by full-time staff members provided by the libraries in which these centers are located. The National Administrative Center (located in Peking University) - overseen by officials from the concerned office at the Ministry of Education and the presidents of Peking and Tsinghua Universities and advised by an advisory committee consisting of experts from major member libraries - is responsible for the construction and management of CALIS, makes policies and regulations, and prepares resource-sharing agreements. The center has an office handling routine management needs and several specialized work groups overseeing CALIS' national projects, such as those for the development of databases for union catalogues, current Chinese periodicals, and CALIS' service software.

Under the guidance of the National Administrative Center, five national information centers are each responsible for building and maintaining an information system 
in one of five general areas-humanities, social science, and science; engineering and technology; agriculture and forestry; medicine; and national defense-in coordination with regional centers and member libraries. The host libraries where these centers are located possess relatively abundant collections in their respective areas. These centers, which are intended to be information bases that cover all major disciplines of science, are responsible for importing databases for sharing and constructing resource-sharing networks among member libraries and for providing searching and document delivery services to member libraries.

Depending on their location, academic libraries in China are divided into eight groups, with each forming a regional library consortium. Each regional consortium is overseen by a regional management center, except for the consortium in the north, which is directly managed by the national management center. The regional centers not only participate in nationwide projects in coordination with the national centers and other regional centers, but they also are responsible for promoting cooperation among libraries in their particular regions.

All the centers are located in member universities and staffed by the host universities. The concerned vice president or library director of a host university is in charge of the associated center. The regional centers also are assisted by regional coordination committees and advisory committees of provincial and municipal officials in charge of education; university presidents; library directors; and senior librarians in the concerned

Table 1.

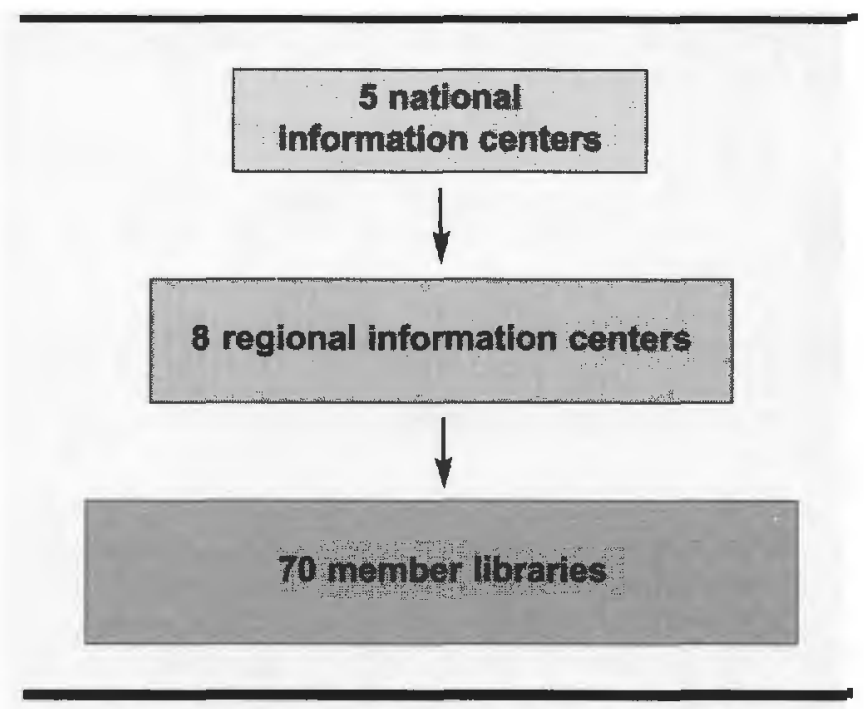

Figure 1. The Three-Tiered Structure of CALIS

Five National Information Centers

\begin{tabular}{ll}
\hline Areas of specialization & Location \\
\hline Humanities, Social Science and Science & Peking University, Beijing \\
Engineering and Technology & Tsinghua University, Beijing \\
Agriculture and Forestry & China Agricultural University, Beijing \\
Medicine & Beijing Medical University, Beijing \\
National Defense & Haerbin Industrial University, \\
& Haerbin, Heilongjiang \\
\hline
\end{tabular}

Table 2.

Regional Information Centers and Areas of Their Jurisdiction

\begin{tabular}{lll}
\hline Name & Location & Areas of juristiction \\
\hline National Administrative Center & Beijing & Beijing, Tianjin, Hebei, Shanxi, and Inner Mongolia \\
Southeast (South) Regional Center & Shanghai & Shanghai, Zhejiang, Fujian, and Jiangxi \\
Southeast (North) Regional Center & Nanjing & Jiangsu, Anhui, and Shandong \\
Central Regional Center & Wuhan & Hubel, Hunan, and Henan \\
South Regional Center & Guanzhou & Guangdong, Hainan, and Guangxi \\
Southwest Regional Center & Chengdu & Sichuan, Chongqing, Yunnan, and Guizhou \\
Northwest Regional Center & Xi'an & Shanxi, Gansu, Ningxia, and Xinjiang \\
Northeast Regional Center & Jilin & Jilin, Liaoning, and Heilongjiang \\
\hline
\end{tabular}


regions. These committees serve a coordinating role in the regions.

\section{Funding}

The development and operation of CALIS has been funded in large part by the Chinese government. The sources of funding for CALIS at the present time are as follows:

- Government grants. Much of the funds for the CALIS project during the first phase of construction came from the government. Because of the demonstrated benefits of the ongoing project, it is expected that the government will provide funds for the second phase of CALIS construction. These government funds have been used in the purchase of software and hardware for the CALIS centers and commercial databases, development of service software and databases, training of staff members, etc.

- Local matching funds. According to prior agreements, a province or city that desires to have a regional center is required to provide funds in supplementation to the government funds for the construction of its local center.

- Member library funds. These funds, primarily derived from the university budgets, have been used to purchase electronic resources and cover the expenses incurred from the use of the CALIS service software platforms.

Although CALIS is currently funded by the government, the future expansion and operation of the system is expected to rely in large part on other sources of funds. The funding needs for CALIS may be met by operating the system in a commercial mode.

\section{Principles for Cooperation among Members}

The successful operation of a library consortium clearly depends on good working relationships among members and between members and the consortium. At CALIS, all members are required to adhere to a set of principles (see below) in dealing with these relationships. It is based on these principles, known as the CALIS Principles for Cooperation among Members, that CALIS policies and rules are made.

- The common interests of CALIS are above those of individual member libraries.
- Member libraries should not cooperate at the expense of the interests of others.

- CALIS provides services to member libraries for no profit.

- Member libraries are all equal and enjoy the same privileges.

- Larger member libraries are obliged to make more contributions.

\section{What Has Been Achieved?}

When it was first established, CALIS had sixty-one member libraries from major universities participating in "Project 211." Later, as many other major universities were interested in joining the alliance, the number of CALIS members has climbed to seventy. At the present, CALIS serves about 700,000 students.

Construction of CALIS is a long-term, strategic undertaking. The system provides service functions as they become available and is constantly being improved in the process. In the first phase (1998 to 2000) of the project, CALIS successfully started the following information-sharing functions in its member libraries:

- primary and secondary data searching;

- interlibrary borrowing and lending;

- document delivery;

- coordinated purchasing; and

- online cataloguing.

The following tasks have been completed:

- purchase of computer hardware (e.g., SUN E3500);

- construction of a CERNet- or Internet-based information-sharing network connecting academic libraries across the country; and

- group purchase of databases, such as UMI, EBSCO, EI Village, INSPEC, Elsevier, and Web of Science, that are shared among member libraries either directly online or indirectly through requested service/document delivery.

CALIS also has completed development of a number of databases, including:

- Union Catalogues. These databases currently contain 500,000 bibliographic records of the Chinese and Western language books and periodicals in all member libraries.

- Dissertation Abstracts and Conference Proceedings. These databases now contain abstracts of doctoral dissertations (12,000 bibliographic records) and proceedings of national and international conferences (8,000 records) collected from more than thirty 
member libraries. The databases are expected to have 40,000 records in total by the end of 2000 .

- Current Chinese Periodicals. These databases 5,000 titles, 1.5 million bibliographic records) contain contents and indexes of current Chinese periodicals from about thirty member libraries.

- Key Disciplines Databases. CALIS has sponsored the development of twenty-five discipline-specific databases by member libraries. Each of these databases contains about 50,000 to 100,000 records.

The first three classes of databases are prepared in the USMARC, UNIMARC, or CCFC format for the ease of use by patrons and cataloguing staff and in data exchange. Clients from member libraries may perform a Web-based search of the above databases. Most of them contain secondary documents and abstracts, and access CALIS online resources using browsers.

Development of software platforms includes the following:

- Cooperative online cataloguing systems. The systems include protocol Z39.50-based search and uploading servers and terminal software platforms for cataloguing staff. Acquisition and cataloguing staff in each member library may participate in cooperative online cataloguing using the terminal software platforms on their local system. The systems have been used for the development and operation of the union catalogue databases.

- Systems for database development. These systems can be used in the development of shared databases containing secondary data information in USMARC, UNIMARC, CCFC, or Dublin Core format. The systems for database development in the USMARC, UNIMARC, or CCFC formats are equipped with a search server based on the Z39.50 protocol to permit use by cataloguing staff and for data exchange.

- An interlibrary loan system. The system, developed based on the ISO10160/10161 protocol, consists of ILL protocol machines and client terminals. These systems, located in member libraries, are interconnected to form a CALIS interlibrary loan network. Primary document delivery software based on the FTP protocol also has been developed for the delivery of scanned documents between libraries.

- An OPAC system. The system has both Web/Z39.50 and Web/ILL gateways. Patrons may visit the system using common browsers, search all CALIS

\section{NEW!}

\section{Getting the Most Out of Web-based Surveys}

by David Ward $=2000$

\$20 (\$18 LITA members)

ISBN 0838981089

Surveys help evaluate user services, rate different library programs, facilitate needs assessments, aid faculty research, and more. Posting surveys to the Web provides an easy and convenient way to reach intended audiences, centralizes data collection and gives librarians greater control over analyzing and reporting results. This guide shows how to create robust Web-based surveys, and then gather and assimilate their data for use in common database and spreadsheet programs. The author has applied the techniques described in his own work and has designed both commercial and academic Web sites.

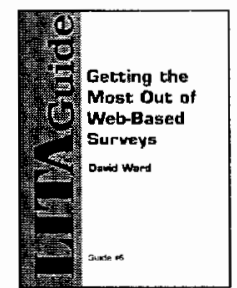

Digital Imaging of Photographs: A Practical Approach to Workflow Design and Project Management

by Lisa Macklin and Sarah Lockmiller 1999 \$20 (\$18 LITA members) - ISBN 0838980058

A comprehensive approach to management of digital imaging in libraries and archives, from archival negatives to metadata cataloging and Web-based access.

\section{Getting Mileage Out of Metadata: Applications for the Library}

by Jean Hudgins, Grace Agnew, and Elizabeth Brown 1999 a \$22 (\$19.20 LITA members) a ISBN 0838980066

An overview of the state-of-the-art metadata cataloging and currently available metadata standards, including comprehensive descriptions and links to current applications.

Other LITA publications and a printable order form can be found at www.lita.org/litapubs/index.html. Fax orders to (312) 836-9958 or call 800-545-2433, press 7 (M-F, 8-5 CST). 
databases, and send search results directly to the CALIS interlibrary loan service. Patrons also may access an ILL server through Web/ILL, tracking the status of submitted interlibrary loan requests, inquiring about fees, and so on.

The databases that are centrally located and those that are distributed at various locations as well as service platforms in member libraries form a CALIS information service network.

\section{Future Considerations}

In a period of just over a year, considerable progress has been made in forming a nationwide resource-sharing library consortium in China. However, because member libraries vary in size, available funds, staff quality, and automation level, CALIS has yet to realize its potential. There are a number of problems that remain to be solved. For example, the CALIS union catalogue databases do not work well on some of the old automation systems in member libraries and the CALIS service platforms are incompatible with a dozen automation systems currently in use; as a result, the union catalogues cannot tell the real-time circulation status in all member libraries, affecting interlibrary loan service. In addition, primary resources are not sufficiently abundant. Therefore, the extent to which resources are shared among member libraries remains quite limited.

In the next phase of development, CALIS will improve service systems (including hardware and software platforms) and the distribution of shared databases. At the same time, CALIS will develop more electronic resource databases and be actively involved in the research and development of digital libraries, expanding the scale and extent of resource sharing.

\section{References}

1. Barbara A. Winters, "Access and Ownership in the 21st Century: Development of Virtual Collection in Consortial Settings," in Electronic Resources and Consortia (Taiwan: Science and Technology Information Center, 1999), 163-80; Katherine A. Perry, "VIVA (The Virtual Library of Virginia): Virtual Management of Information, in Electronic Resources and Consortia (Taiwan: Science and Technology Information Center, 1999), 93-114; Delmus E. Williams, "Living in a Cooperative World: Meeting Local Needs Through OhioLINK," in Electronic Resources and Consortia, Ching-Chin Chen, ed. (Taiwan: Science and Technology Information Center, 1999), 137-61.

2. Jordan M. Scepanski, "Collaborating on New Missions: Library Consortia and the Future of Academic Libraries," in Proceedings of the International Conference on New Missions of Academic Libraries in the 21st Century, Duan Xiaoqing and $\mathrm{He}$ Zhaohui, eds. (Peking: Peking Univ. Pr., 1998), 271-75. 